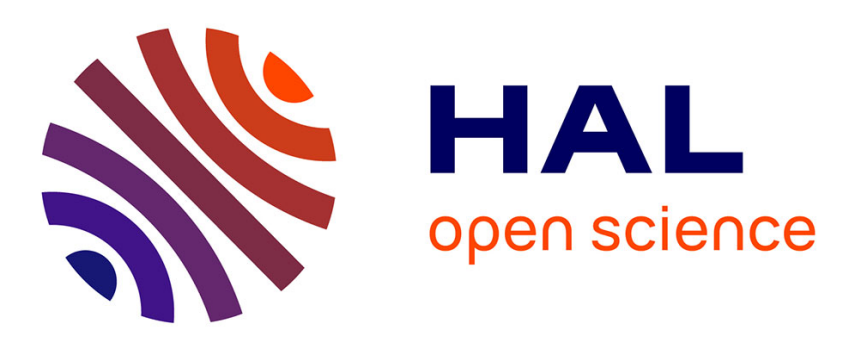

\title{
A Multiscale Approach for Nonlinear Dynamic Response Predictions With Fretting Wear
}

J. Armand, L. Pesaresi, L. Salles, C. Schwingshackl

\section{To cite this version:}

J. Armand, L. Pesaresi, L. Salles, C. Schwingshackl. A Multiscale Approach for Nonlinear Dynamic Response Predictions With Fretting Wear. Journal of Engineering for Gas Turbines and Power, 2017, 139 (2), 10.1115/1.4034344 . hal-02543792

\section{HAL Id: hal-02543792 \\ https://hal.science/hal-02543792}

Submitted on 15 Apr 2020

HAL is a multi-disciplinary open access archive for the deposit and dissemination of scientific research documents, whether they are published or not. The documents may come from teaching and research institutions in France or abroad, or from public or private research centers.
L'archive ouverte pluridisciplinaire HAL, est destinée au dépôt et à la diffusion de documents scientifiques de niveau recherche, publiés ou non, émanant des établissements d'enseignement et de recherche français ou étrangers, des laboratoires publics ou privés. 


\section{A Multiscale Approach for Nonlinear Dynamic Response Predictions With Fretting Wear}

Mechanical Engineering

Imperial College London,

London SW7 2AZ, UK

e-mail: j.armand13@ic.ac.uk

L. Pesaresi

Mechanical Engineering, Imperial College London,

London SW7 2AZ, UK

L. Salles

Mechanical Engineering, Imperial College London,

London SW7 2AZ, UK

\section{W. Schwingshackl}

Mechanical Engineering,

Imperial College London,

London SW7 2AZ, UK

\begin{abstract}
Accurate prediction of the vibration response of aircraft engine assemblies is of great importance when estimating both the performance and the lifetime of their individual components. In the case of underplatform dampers, for example, the motion at the frictional interfaces can lead to a highly nonlinear dynamic response and cause fretting wear at the contact. The latter will change the contact conditions of the interface and consequently impact the nonlinear dynamic response of the entire assembly. Accurate prediction of the nonlinear dynamic response over the lifetime of the assembly must include the impact of fretting wear. A multiscale approach that incorporates wear into the nonlinear dynamic analysis is proposed, and its viability is demonstrated for an underplatform damper system. The nonlinear dynamic response is calculated with a multiharmonic balance approach, and a newly developed semi-analytical contact solver is used to obtain the contact conditions at the blade-damper interface with high accuracy and low computational cost. The calculated contact conditions are used in combination with the energy wear approach to compute the fretting wear at the contact interface. The nonlinear dynamic model of the blade-damper system is then updated with the worn profile and its dynamic response is recomputed. A significant impact of fretting wear on the nonlinear dynamic behavior of the blade-damper system was observed, highlighting the sensitivity of the nonlinear dynamic response to changes at the contact interface. The computational speed and robustness of the adopted multiscale approach are demonstrated.
\end{abstract}

\section{Introduction}

The dynamic response of a system normally occurs at a global level at high frequencies, which makes it a macroscale problem of short duration. By contrast, fretting wear, which is caused by the dynamic response, occurs at asperity level (microscale) over a long period of time. In order to compute the impact of fretting wear on the dynamic response of an assembled structure, the space and time multiscales must be linked together appropriately. A large research effort has focused on modeling fretting wear for a quasi-static problem [1-5], but only a few attempts have been made to investigate the link with the dynamics of a system. Most wear computations are based on finite-element models and solve the contact problem for one cycle, post-process the contact data, and use a wear law, typically Archard's law [6], to calculate wear depth fields. After each cycle the contact surface of the finiteelement (FE) mesh is updated, by moving each node to include the wear depth, and this procedure is repeated until a final number of cycles or a maximum wear depth is obtained. To overcome the prohibitive computational costs of these methods for industrial applications, Gallego et al. [7] have proposed a quasi-static multiscale approach that couples a finite-element model for the global structural behavior to a semi-analytical solver. This allows a much finer discretization of the contact area while keeping computational cost low. Salles et al. [8,9] also used a multiscale approach, but they proposed a numerical treatment of frettingwear under vibratory loading. In their approach, two separate time scales are used: a slow scale for tribological phenomena and a fast scale for dynamics. For a given number of vibration periods, a steady state is assumed and the variables are decomposed into Fourier series. An alternating frequency time (AFT) procedure is performed to calculate the nonlinear forces, after which a hybrid Powell solver is used. This work was the first to use numerical analysis to show the coupling between dynamic and tribological phenomena. They also showed that even with wear depths as small as a few microns, the vibratory response is greatly affected.

In the present paper, a new multiscale approach is proposed to calculate and evaluate the impact of fretting wear on the dynamic behavior of assembled structures. This new approach differs from Ref. [7] in that it applies the semi-analytical method to a nonlinear dynamic problem. The main difference with Salles et al. work lies in the additional use of two separate space scales: a macroscale for the dynamic analysis and a microscale for the contact analysis and wear computation, which allows a better discretization of the contact area and hence a higher accuracy. In the following, each component of the proposed approach is described, and then the approach is applied to an underplatform damper system to demonstrate the impact of wear on the nonlinear dynamic response of the system.

\section{Description of the Multiscale Analysis}

Three separate tools are used for the multiscale analysis: (i) a nonlinear dynamic solver providing a multiharmonic vibration response of the blade-damper system, (ii) a semi-analytical contact solver enabling a refined contact analysis at the blade-damper interface, and (iii) a wear energy approach to calculate the fretting wear of the contacting surfaces. The full approach is summarized in a flow chart (Fig. 3).

Nonlinear Dynamic Analysis. The in-house code, forced response suite (FORSE) is used here to analyze the nonlinear response of the underplatform damper system. FORSE is based on the multiharmonic representation of the steady-state response and allows large-scale realistic friction interface modeling. The main features of the methodology can be found in Refs. [10-14]; in this paper, only an overview of the analysis is presented. The equation 
of motion of an underplatform damper system consists of a linear part, which is independent of the vibration amplitudes, and a nonlinear part resulting from friction at the blade-damper interfaces. It can be written in the following form:

$$
\mathbf{M} \ddot{\mathbf{q}}(t)+\mathbf{C} \dot{\mathbf{q}}(t)+\mathbf{K q}(t)+\mathbf{f}[\mathbf{q}(t), \dot{\mathbf{q}}(t)]=\mathbf{p}(t)
$$

where $\mathbf{q}(t)$ is a vector of displacements for all degrees-of-freedom (DOFs) in the blade-damper system; $\mathbf{K}, \mathbf{C}$, and $\mathbf{M}$ are stiffness, damping, and mass matrices of the linear model, respectively; $\mathbf{f}[\mathbf{q}(t), \dot{\mathbf{q}}(t)]$ is a vector of nonlinear, friction interface forces, which is dependent on displacements and velocities of the interacting nodes; and $\mathbf{p}(t)$ is a vector of periodic exciting forces. The variation of the displacements in time is represented by a restricted Fourier series, which can contain as many harmonic components as necessary to approximate the solution, i.e.

$$
\mathbf{q}(t)=\mathbf{Q}_{0}+\sum_{j=1}^{n}\left(\mathbf{Q}_{j}^{c} \cos m_{j} \omega t+\mathbf{Q}_{j}^{s} \sin m_{j} \omega t\right)
$$

where $\mathbf{Q}_{j}^{c, s}$ are vectors of harmonic coefficients for the system degrees-of-freedom (DOFs); $n$ is the number of harmonics that are used in the multiharmonic displacement representation; and $\omega$ is the principal vibration frequency. The flow chart of the calculations performed with the code is presented in Fig. 1. The contact interface elements developed in Ref. [12] are used to model the nonlinear interactions at contact interfaces and analytical expressions for the multiharmonic representation of the nonlinear contact forces and stiffnesses. The nonlinear algebraic system of the reduced model is obtained using a hybrid reduction method developed by Petrov [14,15], leading to a nonlinear system in the frequency domain

$$
\tilde{\mathbf{Q}}=A(\omega)\left(\tilde{\mathbf{F}}-\tilde{\mathbf{F}}_{\mathrm{nl}}(\tilde{\mathbf{Q}})\right)
$$

where $\tilde{\mathbf{Q}}$ is the vector of the Fourier coefficients of the displacements at the interface, $A(\omega)$ is the frequency response, $\tilde{\mathbf{F}}$ is the vector of the Fourier coefficients of the excitation force, and $\tilde{\mathbf{F}}_{n l}$ is the vector of the Fourier coefficients of the nonlinear contact forces. This nonlinear system is solved by means of the alternating frequency time procedure (AFT) $[9,16]$ depicted in Fig. 2. $\mathbf{z}$ denotes the tangential slips and $\mathbf{w}$ are the wear depths. DFT and iDFT refer to the discrete Fourier transform and the inverse

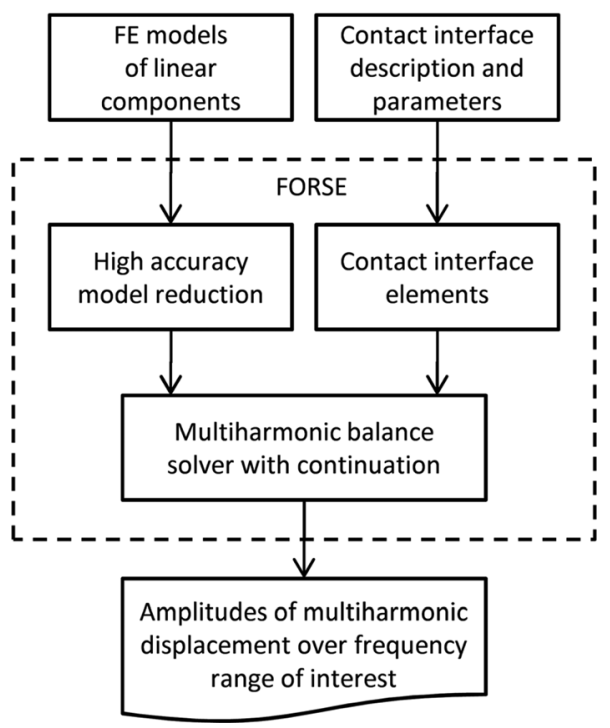

Fig. 1 Scheme of the forced response analysis

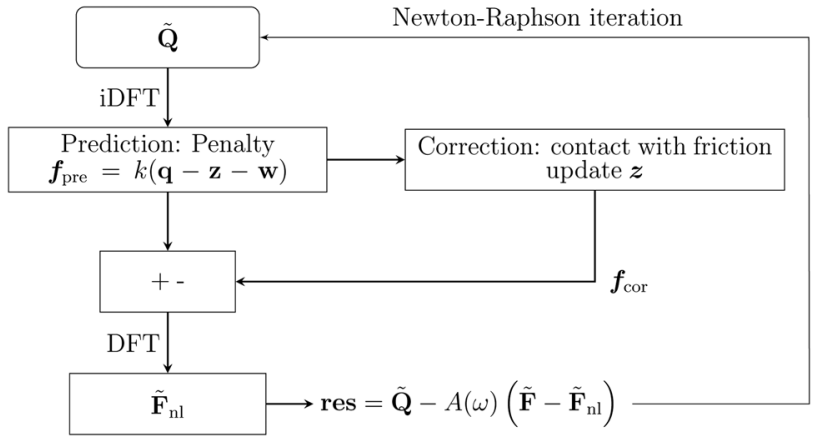

Fig. 2 Scheme of the AFT procedure

discrete Fourier transform, respectively. $f_{\text {pre }}$ is the prediction of the nonlinear contact forces in time domain based on a penalty method and $\boldsymbol{f}_{\text {cor }}$ is the correction of these contact forces to ensure that the frictional contact laws are respected.

Semi-Analytical Contact Solver. The semi-analytical contact solver used for the computation of the refined contact analysis at the interface between the blades and the damper has been presented in detail and validated previously in Refs. [17]. It is used to calculate the initial static normal load distribution required to obtain the reference forced response of the unworn system. In addition, it is used in combination with the wear energy approach to predict the fretting wear of the contacting surfaces over time.

The contact solver is based on the use of the projected conjugate gradient method [18] and a discrete-convolution fast Fourier transform to accelerate the computation. It assumes the elastic half-space body description, which makes it possible to use the Boussinesq and Cerruti potentials $[19,20]$ to compute the elastic deflections in the normal and tangential directions from the pressures and shear tractions in the contact area. Equation (4) gives the component of normal displacement $u_{z}$ due to a pressure distribution $p$. Equation (5) is the discretized form of Eq. (4) on a regular grid of $N_{x} \times N_{y}$ points. Similar equations are used to compute the tangential displacements. The first step of the algorithm is to solve the normal contact problem using the conjugate gradient method, after which the tangential problem can be solved using the Coulomb friction law to bind the shear distribution in the slipping region

$$
u_{z}(x, y)=\frac{1-\nu^{2}}{\pi E} \int_{-\infty}^{+\infty} \int_{-\infty}^{+\infty} \frac{p(\xi, \eta)}{\sqrt{(\xi-x)^{2}+(\eta-y)^{2}}} d \xi d \eta
$$

where $E$ and $\nu$ are the Young's modulus and Poisson ratio of the material, respectively

$$
u_{z}(i, j)=K_{z z} \otimes p=\sum_{k=1}^{N_{x}} \sum_{l=1}^{N_{y}} p(k, l) K_{z z}(i-k, j-l)
$$

where $\otimes$ denotes the discrete convolution product and $K_{z z}(i, j)$ are the discrete influence coefficients that give the normal displacement resulting from unit pressure on the element centered on the grid point $(i, j)$.

Wear Computation. Once the normal and tangential contact conditions have been calculated with the semi-analytical solver, the wear energy approach [2,21-23] is used to compute fretting wear. In this approach, the total wear volume obtained after $N_{\text {cycles }}$ is assumed to be proportional to the accumulated dissipated energy 


$$
V=\alpha \sum_{n=1}^{N_{\text {cycles }}} E d_{n}
$$

where $\alpha$ is a wear coefficient determined experimentally and $E d_{n}$ is the dissipated energy due to friction during the $n$th cycle.

Based on the wear coefficient, the wear depth $\Delta h_{i j}$ at each node after one cycle can be calculated using

$$
\Delta h_{i j}=\alpha \int_{0}^{T}\left\|\mathbf{q}_{i j}(t)\right\|\left\|\Delta \mathbf{v}_{i j}(t)\right\| d t
$$

where $\mathbf{q}_{i j}$ and $\Delta \mathbf{v}_{i j}$ are, respectively, the vectors of the two tangential shear stresses and the relative tangential velocities at the grid point $(i, j)$, and $T$ is the duration of one vibration cycle. The energy wear coefficient used in the analysis is based on Leonard [24] and is equal to $2 \times 10^{3} \mu \mathrm{m}^{3} \mathrm{~J}^{-1}$ for a steel-steel fretting contact; however, it should be noted that for an accurate analysis, proper fretting wear measurements should be performed on the used materials in order to obtain an accurate energy wear coefficient.

Proposed Multiscale Approach. The multiscale approach is summarized in the flow chart in Fig. 3. First of all, a refined contact analysis is performed using the semi-analytical contact solver to provide the static pressure and gap distributions required for the definition of the 3D contact elements used in FORSE. Then, a nonlinear dynamic analysis is conducted in FORSE to obtain the unworn nonlinear dynamic response and the contact loads (forces and moments) at the interface. The sum of the contact loads are then computed at the center of the contact area over a vibration cycle, including the normal force $N$, the two tangential forces $T_{x}$ and $T_{y}$, the two bending moments $M_{x}$ and $M_{y}$, and the torsional moment $M_{z}$. The resulting forces and moments are used in the SA contact solver to determine the pressure, shear, and slip fields for each increment of the fretting cycle. The convergence of the solution for each increment is ensured by enforcing the residuals of the conjugate gradient to be minimum within a given tolerance. The wear energy approach is then used to calculate the wear depth over the contact surface. The wear obtained after one vibration cycle is so small that it would not impact the interface forces, and therefore a number of cycles $N_{\text {cycles }}$, associated with a total wear depth of $\Delta h_{\max }$, is computed before the refined contact analysis is performed again (referred to as a "wear iteration"). The nonlinear dynamic model is updated at a lower frequency which can be based on a given number of wear iterations or on the total wear volume. The 3D nonlinear contact element definition is updated with the new worn profile and the corresponding new static pressure distribution obtained from the SA solver, and a new nonlinear dynamic response is computed, which provides an updated set of contact forces to the wear computation. This procedure is continued until either a maximum total number of cycles or a maximum total wear volume at the contact interface has been reached.

\section{The Underplatform Damper Test Case}

The test case selected to demonstrate the developed analysis technique is a blade-damper system, which is a very common mechanical structure in high-pressure turbines (HPT) with a wellunderstood nonlinear dynamic behavior. Past experience indicates that significant wear can occur at the damper during operation, but its impact on the bladed-disk dynamics is normally neglected. A simplified blade-damper geometry of a recently developed underplatform damper test rig [25] will be used to demonstrate the change in the nonlinear dynamic response due to damper wear.

Linear Finite-Element Model. Using the Rolls-Royce inhouse finite-element (FE) code SC03, a full three-dimensional finite-element model, depicted in Fig. 4, has been generated with quadratic hexahedral elements for both blades (54,972 elements) and the damper (3620 elements). Particular care has been taken to refine the mesh at the contact interfaces to allow the introduction of a sufficient number of friction elements for the nonlinear analysis. This fine discretization of the contact is required to capture local microslip effects which play a critical role in the wear process. The wear analysis will focus on the first out-of-phase flap mode of the two blades (see Fig. 4), which shows no damper rotation and therefore maximizes the dissipated energy and wear at the contact interfaces.

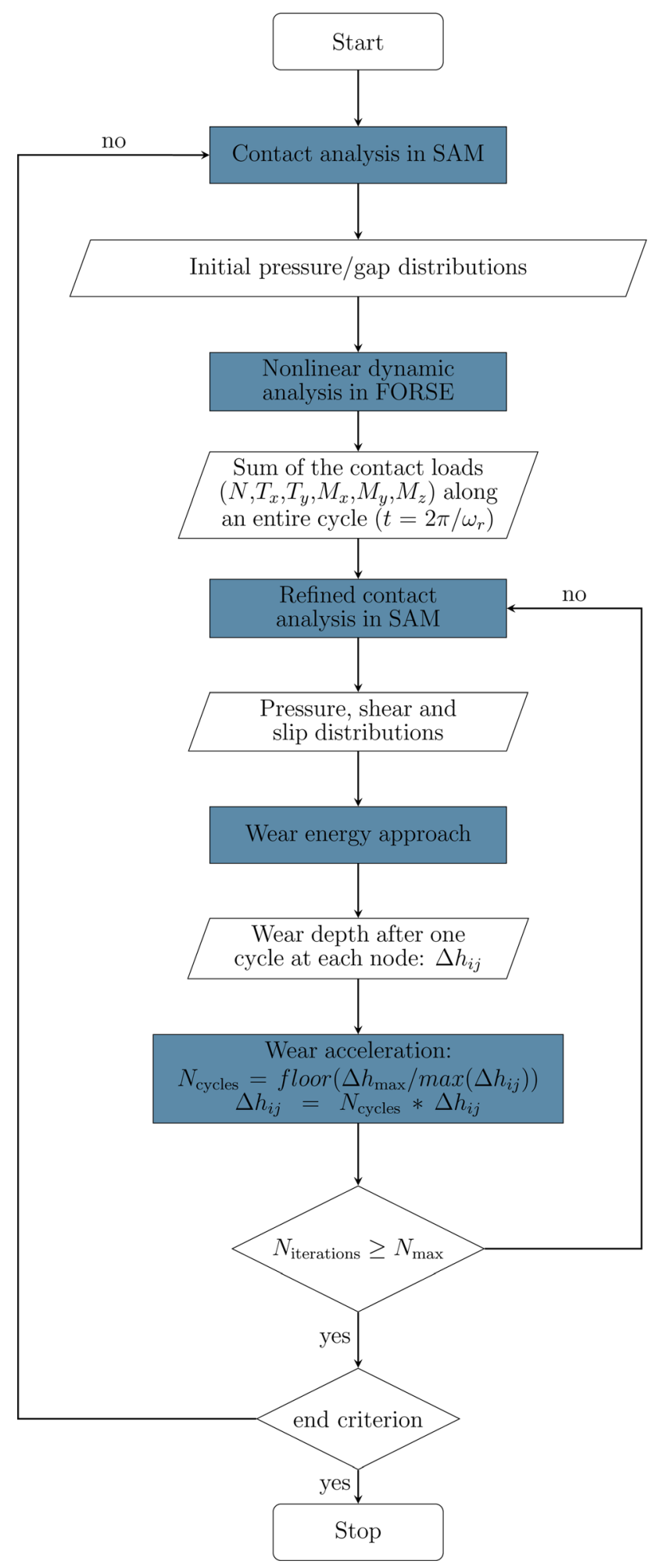

Fig. 3 Scheme of the multiscale analysis 


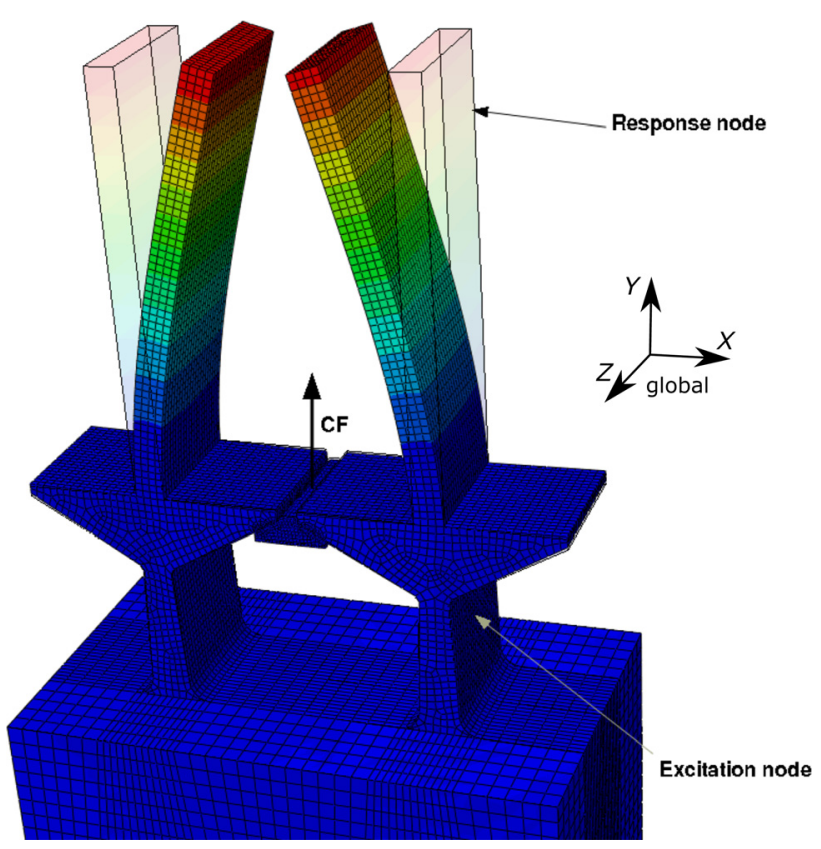

Fig. 4 First out-of-phase flap mode of the two blades

Nonlinear Contact Model. The coupling between the blades and the damper is achieved by 152 full three-dimensional friction elements at each blade-damper interface, as shown in Fig. 5. The chosen number of elements enables a good discretization of the contact area, while keeping the computational time acceptable. Each friction element connects a linear finite-element node from one side of the contact to its matching node on the opposing side of the contact and transmits the nonlinear forces. The friction element, which is depicted in Fig. 6, is defined by the area $A$ it covers, the friction coefficient $\mu$, the tangential and normal contact stiffness $k_{t}$ and $k_{n}$, and the static pressure $p$, which describes the initial contact condition at the blade-damper interface. It is also possible to account for an initial gap $g$ by specifying a negative initial pressure $p=-k_{n} * g / A$. This last feature will be used during the analysis to update the nonlinear dynamic model and include the worn geometry during the iterations, thereby eliminating the need for updating and recalculation of the linear finiteelement model.

\section{Results}

Static Normal Load. The semi-analytical contact solver was used to solve the normal contact problem between the damper and the blade; this provides the accurate static normal load required to perform the initial nonlinear dynamic analysis. The input parameters for this computation are given in Table 1 . The normal load on each contact patch is derived from the centrifugal load

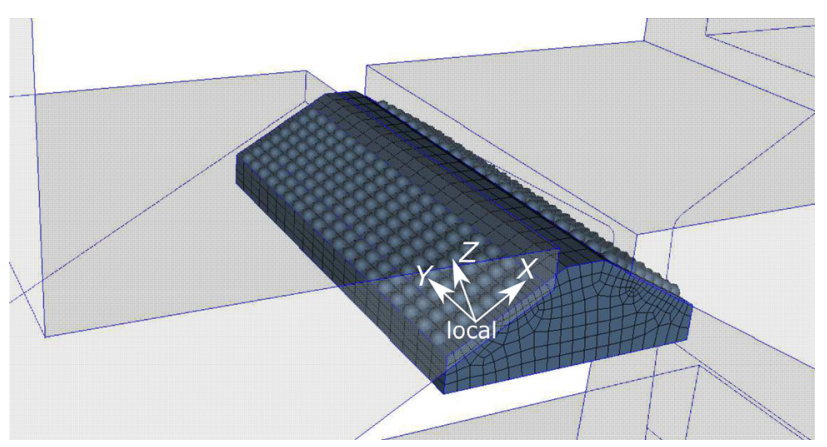

Fig. 5 Nonlinear contact element locations

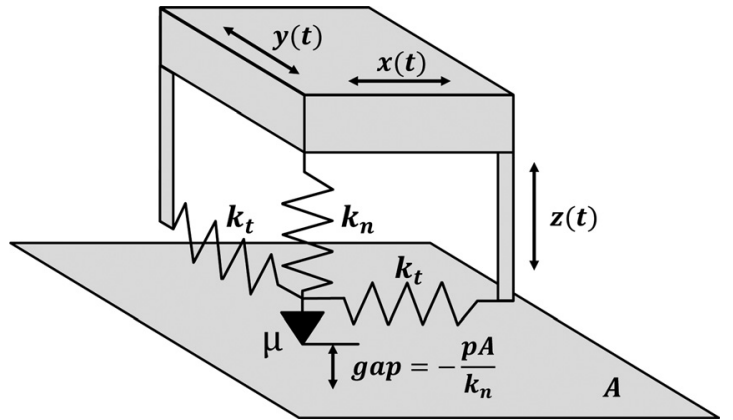

Fig. 6 Friction interface element

Table 1 Calculation parameters

\begin{tabular}{ll}
\hline \hline Parameter & Value \\
\hline Young's modulus of the blade, $E_{1}(\mathrm{GPa})$ & 210 \\
Young's modulus of the damper, $E_{2}(\mathrm{GPa})$ & 210 \\
Poisson ratio of the blade, $\nu_{1}$ & 0.3 \\
Poisson ratio of the damper, $\nu_{2}$ & 0.3 \\
Friction coefficient, $\mu$ & 0.6 \\
Normal load, $N(\mathrm{~N})$ & 428.81 \\
\hline
\end{tabular}

$C F=1 \times 10^{3} \mathrm{~N}$, applied to the damper, as shown in Fig. 4, using the following formula:

$$
N=\frac{1}{2} \frac{C F}{(\cos (\theta)+\mu \sin (\theta))}
$$

where $\theta$ is the damper angle and $\mu$ is the friction coefficient.

Each face of the blade-damper contact interface is discretized into a regular grid of $128 \times 128$ elements for the SA analysis. The contacting surfaces are assumed to be perfectly flat and smooth such that there is no initial separation between the damper and the blade. The calculated pressure distribution, presented in Fig. 7, led to a uniform central part and exhibited higher values along the boundaries of the contact area, which is expected given the singularity of the stress fields at these locations. It should also be borne in mind that the semi-analytical contact solver is based on the half-space assumption, which may lead to a slight loss of accuracy. From this very detailed pressure distribution, the value at each nonlinear contact node was linearly interpolated to provide the initial static pressure required for the nonlinear dynamic

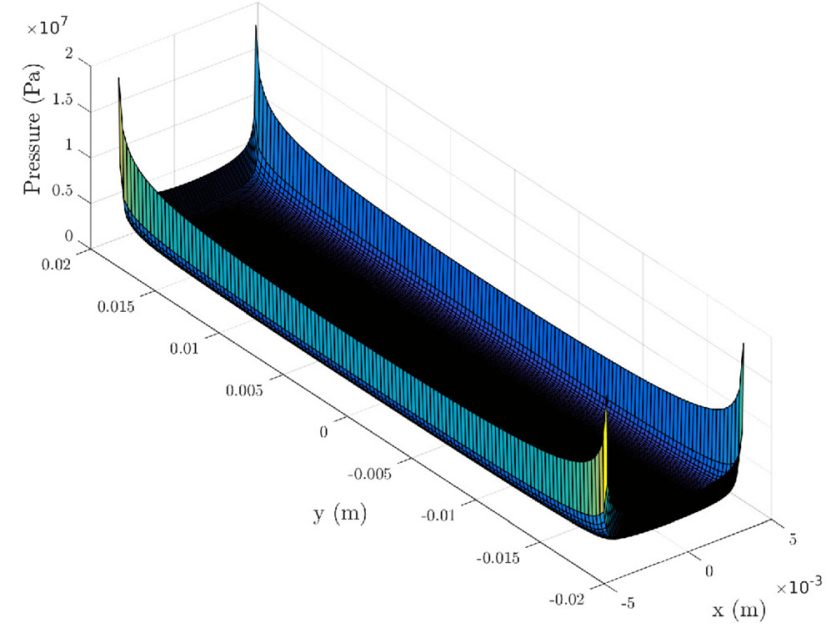

Fig. 7 Initial pressure distribution 
analysis in FORSE. None of the nonlinear nodes is located on the boundaries of the contact area, which mitigates the impact of the boundary effects inherent to the flat-on-flat contact geometry.

Nonlinear Dynamic Behavior of the Unworn System. An initial nonlinear dynamic analysis was performed on the unworn system. The excitation and response node locations are shown in Fig. 4; the excitation and response are in the $X$ direction of the global coordinate system shown Fig. 4 . Figure 8 shows the nonlinear frequency response functions of the first out-of-phase flap mode of the unworn blade-damper system under a very low excitation level $(0.1 \mathrm{~N})$ that leads to a linear behavior and under a higher excitation $(17 \mathrm{~N})$ for which a strong nonlinear behavior can be observed. The nonlinear behavior is due to the transition of some of the frictional elements in the contact area from stick to slip at resonance. This behavior is illustrated in Fig. 9, which shows the state of the nonlinear contact elements at resonance under the $17 \mathrm{~N}$ excitation. It can be seen that the lower parts of the damper remain fully stuck, while the rest of the elements experience stick-slip transitions. A line of contact elements (nodes labeled "Separation-Stick-Slip" in Fig. 9) near the upper edge of the patch even experiences separation during the vibration cycle. This separation is due to the widening of the upper gap between the platforms during a vibration cycle. Figure 10 shows the normal and tangential force fields at resonance at the point in the vibration cycle when the total tangential force is maximum. It can be seen that the tangential forces at the nodes $F_{\mathrm{t}}$ are mostly orientated in the $x$ direction (of the local coordinate system shown Fig. 5 ) and that the normal load $F_{\mathrm{n}}$ shows a gradient along the $x$ direction, which results in the moment $M_{y}$ shown in Fig. 11. These results are consistent with the expected physical behavior of the blade-damper system; for the first out-of-phase flap mode, the damper is moving straight up and down, leading to only one component of tangential forces $\left(F_{x}\right)$ combined with the normal force $F_{n}$. Furthermore, when the damper experiences this pure translation, a relative rotation around an off-centered line between the two contact surfaces generates a moment $M_{y}$, as can be observed in the vector plot of Fig. 10. The moments $M_{y}$ on the right and left side of the damper are equal and opposite so that they have no effect on the damper motion. As previously discussed, the semianalytical contact solver requires the forces and moments at the center of the contact patches as inputs. Figure 12 shows the sum of the nodal forces on the left contact patch during one vibration cycle at resonance for a $17 \mathrm{~N}$ excitation, and Fig. 11 shows the sum of the moments. The resulting total loads are nearly identical between the right and left contact patches due to the symmetry of the system and of the considered mode.

Refined Contact Analysis and Wear Computations. Following the computation of the nonlinear forces at the center of the contact interfaces, these values are used with the semi-analytical contact solver to obtain more accurate contact conditions for the wear calculation. For this analysis, the input parameters were the

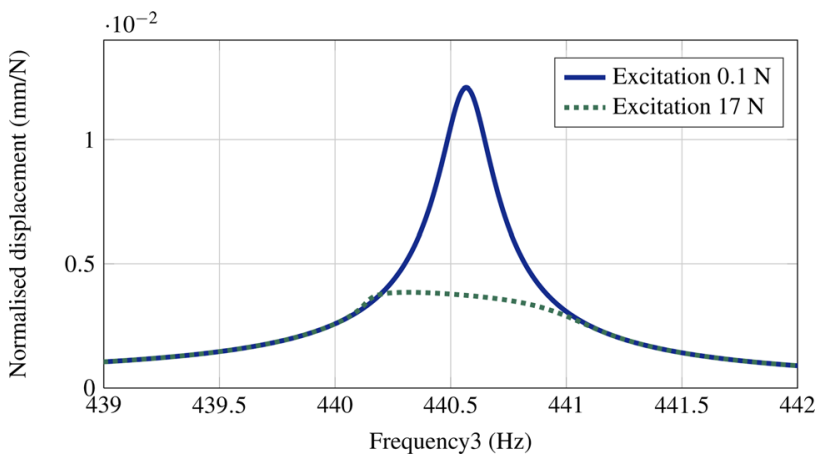

Fig. 8 Frequency response function of the unworn system

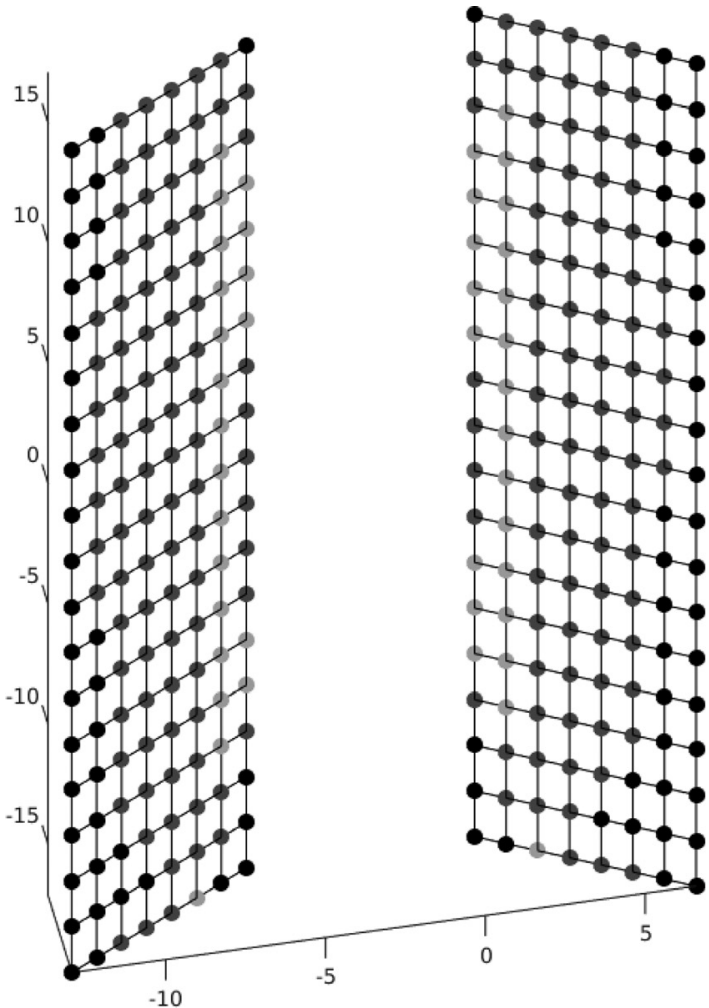

- Full Stick (80 nodes)

- Stick/Slip (180 nodes)

- Separation-Stick-Slip (44 nodes) Full separation (0 nodes)

Fig. 9 Contact conditions at resonance

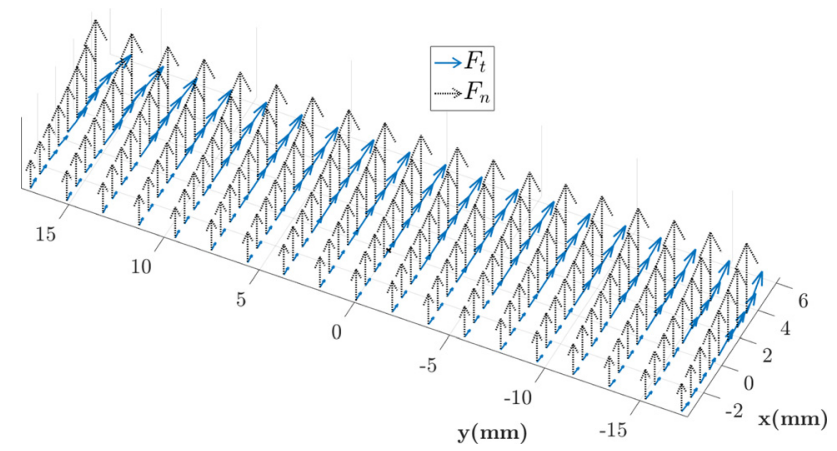

Fig. 10 Normal and tangential force fields at resonance

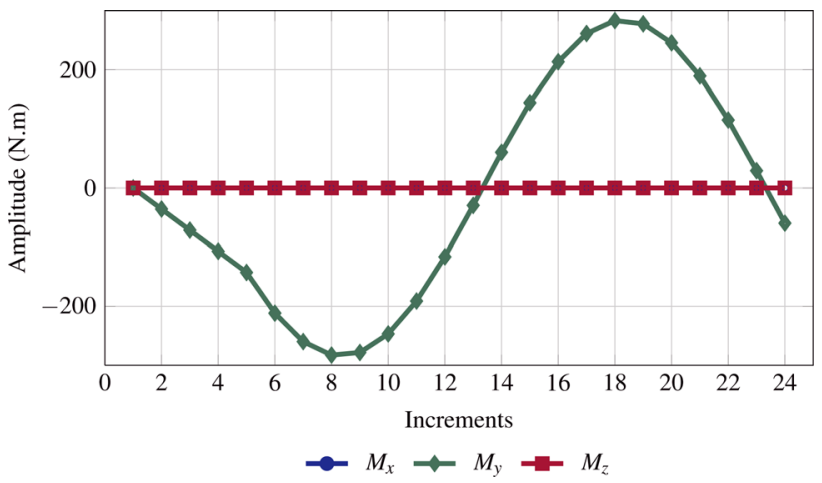

Fig. 11 Total moments at the center of the left contact patch 


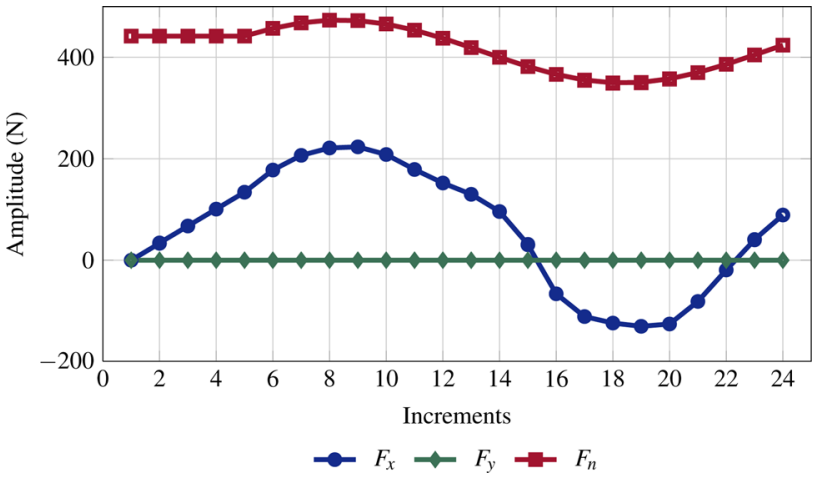

Fig. 12 Total forces at the center of the left contact patch

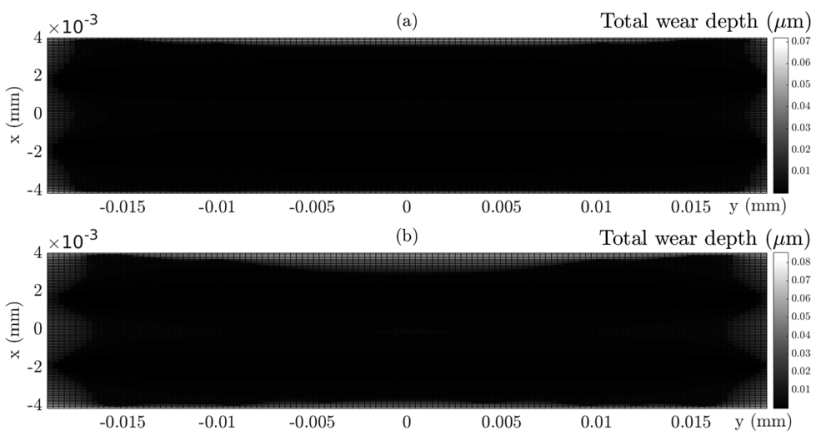

Fig. 13 Wear patterns after $500(a)$ and $1000(b)$ iterations

same as those presented in Table 1 . The results are used in conjunction with the wear energy approach to compute the fretting wear at the contact interface. Figure 13 shows the total wear depth obtained after 500 and 1000 wear iterations (which corresponds to approximately $5.7 \times 10^{9}$ and $1.1 \times 10^{10}$ vibration cycles). It can be seen that the wear is concentrated along the four edges of the contact patch, where the moment $M_{y}$ around the $y$-axis leads to a slightly asymmetric pressure and more wear on the top edge of the contact patch. Figure 14 displays the evolution of the pressure distribution with wear. Once the edges of the contact patch are worn out, the pressure distribution is no longer singular at these locations and bounded peaks are now observed at the boundaries of the newly worn contact patch. This redistribution of the pressure leads to a slight increase at the center of the contact interface, creating a new initial condition for the nonlinear dynamic analysis. These results were obtained using MATLAB on a regular workstation (Intel Core i3-4340 3.6 GHz); each wear iteration took approximately $80 \mathrm{~s}$ (wall clock time).

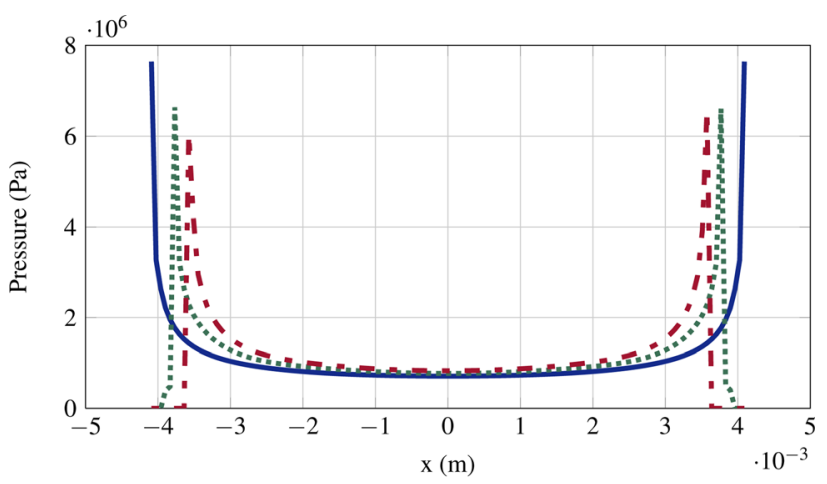

$$
\begin{aligned}
& \text { - Unworn profile } \\
& \text { - } \quad \text { Worn profile } 1000 \text { iterations }
\end{aligned}
$$

Fig. 14 Impact of wear on pressure distribution

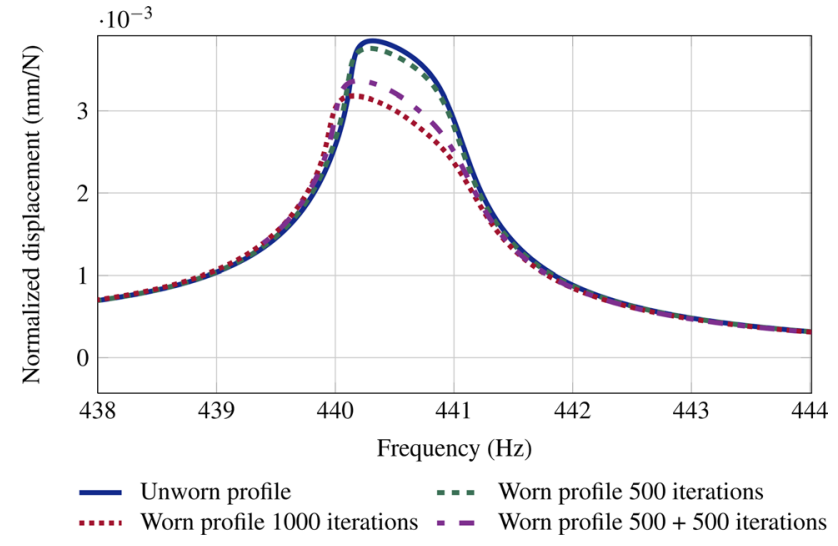

Fig. 15 Impact of wear on the nonlinear FRF

Impact of Wear on the Nonlinear Dynamic Response. Figure 15 shows the nonlinear FRFs obtained for three different worn configurations: for the first two configurations, the nonlinear dynamic model is updated only once after 500 and 1000 wear iterations. For the third configuration, the nonlinear dynamic model is updated every 500 iterations. It is very interesting to see that even with a wear scar covering only a small area and a maximum local wear depth as small as $0.07 \mu \mathrm{m}$, a significant impact on the nonlinear response of the system can be observed, both in terms of damping and resonance frequency. A notable difference can be seen between the response obtained after updating the nonlinear dynamic model every 500 and 1000 iterations, which indicates

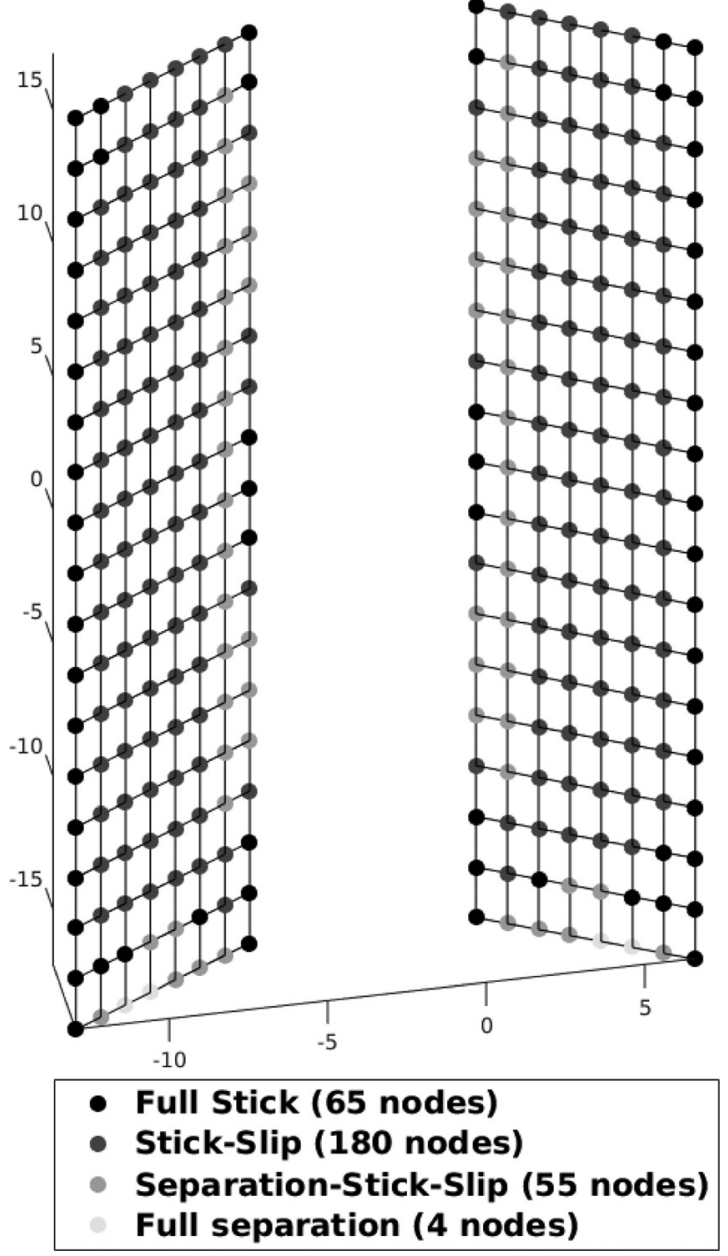

Fig. 16 Contact conditions at resonance with the worn profile 


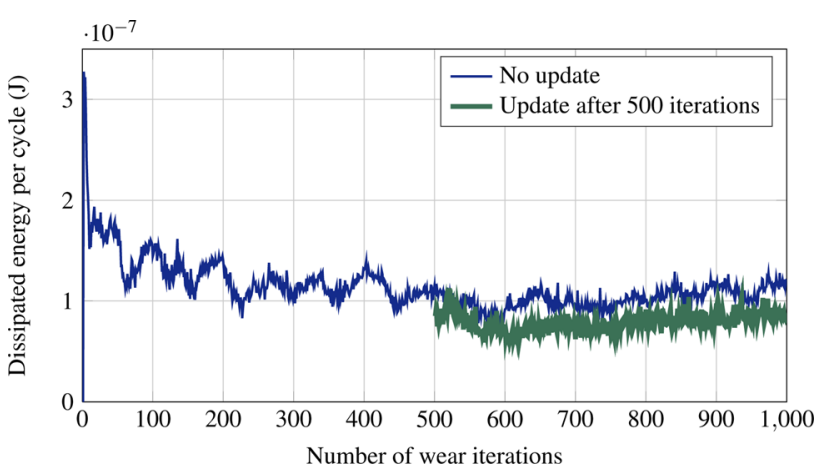

Fig. 17 Evolution of the dissipated energy per cycle over time

that the nonlinear dynamic model should be updated more often. As previously discussed, updating the nonlinear dynamic model after every wear iteration would not be sound because the amount of wear at each iteration is so small that it would result in no noticeable impact on the dynamic response. Furthermore, the computational cost of such a process would be prohibitive, which is why an optimum number of wear iterations should be computed before updating the nonlinear dynamic model. These simulations were run on a cluster node with a 20-core CPU Intel Xeon E5-2620 $2 \mathrm{GHz}$ and lasted for approximately $5 \mathrm{~h} 45 \mathrm{~min}$ each (wall clock time).

Figure 16 shows the contact conditions at resonance obtained with the worn profile after 1000 wear iterations. The lower pressure at the edges of the contact area, as seen in Fig. 14, results in more nodes experiencing separation and stick-slip transitions during the vibration cycle, which, in turn, leads to a lower resonance frequency and a larger dissipation. Figure 17 shows the evolution of the dissipated energy per cycle, which is proportional to the wear rate over time. It can be seen that after an initial peak, which is due to the no wear/wear condition transition, the wear rate is fairly constant up to the simulated 1000 wear iterations. It can also be observed that updating the nonlinear dynamic model after 500 iterations leads to a lower dissipation, which explains the higher amplitude in the response shown Fig. 15.

\section{Conclusions}

A multiscale approach is proposed to evaluate fretting wear and predict its impact on the nonlinear dynamic response of a blade-damper system. A combination of a multiharmonic balance solver, a newly developed semi-analytical contact solver, and the energy wear approach allowed an accurate and computationally efficient contact analysis and wear computation. The presented results demonstrate the sensitivity of the nonlinear dynamic response to changes at the contact interface; the wear patterns led to a redistribution of the contact pressure, which, in turn, changed the underlying nonlinear mechanisms at the interface from sliding at the edges only to sliding over a larger part of the contact interface. In summary, the proposed multiscale nonlinear dynamic analysis taking wear into account is a powerful tool to accurately predict the nonlinear dynamic response of an assembled structure over its lifetime.

\section{Acknowledgment}

The authors are grateful to Innovate UK and Rolls-Royce plc for providing the financial support for this work and for giving permission to publish it. This work is part of a collaborative R\&T project 'SILOET II Project 10' which is co-funded by Innovate UK and Rolls-Royce plc and carried out by Rolls-Royce plc and the Vibration UTC at Imperial College London.

\section{References}

[1] Fouvry, S., Duó, P., and Perruchaut, P., 2004, "A Quantitative Approach of Ti-6Al-4V Fretting Damage: Friction, Wear and Crack Nucleation," Wear, 257(9-10), pp. 916-929.

[2] Paulin, C., Fouvry, S., and Deyber, S., 2005, "Wear Kinetics of Ti6Al4V Under Constant and Variable Fretting Sliding Conditions," Wear, 259(16), pp. 292-299.

[3] Põdra, P., and Andersson, S., 1999, "Simulating Sliding Wear With Finite Element Method," Tribol. Int., 32(2), pp. 71-81.

[4] McColl, I. R., Ding, J., and Leen, S. B., 2004, "Finite Element Simulation and Experimental Validation of Fretting Wear," Wear, 256(11-12), pp. 1114-1127.

[5] Gallego, L., Nélias, D., and Jacq, C., 2006, "A Comprehensive Method to Predict Wear and to Define the Optimum Geometry of Fretting Surfaces," ASME J. Tribol., 128(3), p. 476.

[6] Archard, J. F., 1953, "Contact and Rubbing of Flat Surfaces," J. Appl. Phys., 24(8), p. 981.

[7] Gallego, L., Fulleringer, B., Deyber, S., and Nélias, D., 2010, "Multiscale Computation of Fretting Wear at the Blade/Disk Interface," Tribol. Int., 43(4), pp. 708-718.

[8] Salles, L., Blanc, L., Thouverez, F., Gouskov, A. M., and Jean, P., 2010, "Multiscale Analysis of Fretting-Wear Under Dynamical Loading," Méc. Ind., 11(3-4), pp. 277-282.

[9] Salles, L., Blanc, L., Thouverez, F., and Gouskov, A. M., 2011, "Dynamic Analysis of Fretting-Wear in Friction Contact Interfaces," Int. J. Solids Struct., 48(10), pp. 1513-1524.

[10] Petrov, E. P., and Ewins, D. J., 2004, "Generic Friction Models for TimeDomain Vibration Analysis of Bladed Disks," ASME J. Turbomach., 126(1), p. 184.

[11] Schwingshackl, C., Petrov, E., and Ewins, D., 2012, "Measured and Estimated Friction Interface Parameters in a Nonlinear Dynamic Analysis," Mech. Syst. Signal Process., 28, pp. 574-584.

[12] Petrov, E. P., and Ewins, D. J., 2003, "Analytical Formulation of Friction Interface Elements for Analysis of Nonlinear Multi-Harmonic Vibrations of Bladed Disks," ASME J. Turbomach., 125(2), p. 364.

[13] Petrov, E. P., and Ewins, D. J., 2004, "State-of-the-Art Dynamic Analysis for Non-Linear Gas Turbine Structures," Proc. Inst. Mech. Eng. Part G, 218(3), pp. 199-211.

[14] Petrov, E. P., 2011, "A High-Accuracy Model Reduction for Analysis of Nonlinear Vibrations in Structures With Contact Interfaces," ASME J. Eng. Gas Turbines Power, 133(10), p. 102503.

[15] Petrov, E. P., and Ewins, D. J., 2007, “Advanced Modeling of Underplatform Friction Dampers for Analysis of Bladed Disk Vibration," ASME J. Turbomach., 129(1), p. 143.

[16] Salles, L., Blanc, L., Thouverez, F., Gouskov, A. M., and Jean, P., 2012. "Dual Time Stepping Algorithms With the High Order Harmonic Balance Method for Contact Interfaces With Fretting-Wear," ASME J. Eng. Gas Turbines Power, 134(3), p. 032503.

[17] Armand, J., Salles, L., and Schwingshackl, C. W., 2015, "Numerical Simulation of Partial Slip Contact Using a Semi-Analytical Method," ASME Paper No. DETC2015-46464.

[18] Polonsky, I. A., and Keer, L. M., 2000, "Fast Methods for Solving Rough Contact Problems: A Comparative Study," ASME J. Tribol., 122(1), p. 36.

[19] Johnson, K. L., 1985, Contact Mechanics, Cambridge University Press, West Nyack, NY, pp. 45-106.

[20] Love, A. E. H., 1906, A Treatise on the Mathematical Theory of Elasticity, Dover, Mineola, NY, pp. 180-200, 217-235.

[21] Huq, M. Z., and Celis, J.-P., 2002, "Expressing Wear Rate in Sliding Contacts Based on Dissipated Energy," Wear, 252(5-6), pp. 375-383.

[22] Fouvry, S., Liskiewicz, T., Kapsa, P., Hannel, S., and Sauger, E., 2003, “An Energy Description of Wear Mechanisms and Its Applications to Oscillating Sliding Contacts," Wear, 255(1-6), pp. 287-298.

[23] Ramalho, A., and Miranda, J., 2006, "The Relationship Between Wear and Dissipated Energy in Sliding Systems," Wear, 260(4-5), pp. 361-367.

[24] Leonard, B. D., 2008, "An Experimental and Numerical Investigation of the Effect of Coatings and Third Body on Fretting Wear," Ph.D. thesis, Purdue University, Lafayette, IN.

[25] Pesaresi, L., Schwingshackl, C. W., Salles, L., Elliot, R., Jones, A., and Green, J. S., 2015, "Numerical and Experimental Investigation of an Underplatform Damper Test Rig," Appl. Mech. Mater., 849, pp. 1-12. 